\title{
Design and evaluation of (urban) camouflage
}

\author{
Maarten A. Hogervorst", Alexander Toet, Pieter Jacobs \\ TNO Defense \& Security, P.O. Box 23, 3769 ZG Soesterberg, the Netherlands
}

\begin{abstract}
An international group consisting of several NATO nations participated in a trial in which urban camouflage was developed and compared. First, photographs were taken in and around a small town (arid climate). Next, the different groups derived urban camouflage patterns from these photographs. We applied our method for deriving a camouflage pattern from a set of (characteristic) background images to the imagery. Eleven different patterns were made into prototype camouflage suits. Panoramic images of all prototype suits were taken on 36 locations in the environment in which the background images were taken. We used these images in a search experiment with human observers for evaluating camouflage performance of the various patterns. The pattern we developed featured among the two best performing patterns, with good performance indicated by low chance of detection and long search times. The results show that our method for deriving a camouflage pattern from background imagery works well.
\end{abstract}

Keywords: visual camouflage, camouflage design, evaluation, human performance, search, conspicuity

\section{INTRODUCTION}

There is currently a lot of interest in the development and evaluation of improved personal camouflage. Historically, the emphasis lay on using a design that displayed strength, was well recognizable and could be discriminated from other troops (e.g. the French wore bright red trousers and blue Greatcoats as part of the standard uniform until into the first World War). Later on, camouflage performance was recognized as an important (additional) feature. Traditionally the design was (and still is) largely based on intuition and aesthetics performed by industrial designers and (especially in the beginning) by artists (incl. cubists such as Georges Braque). The design principles are inspired by the natural world and are based on biological principles as blending and disruption ${ }^{1-3}$.

More recently, scientific studies into the statistics of natural images, texture, visual perception and psychology ${ }^{4}$ have entered the design process. Various camouflage patterns were developed for a range of theatres, including Woodland, Jungle, Desert, Arctic. Two trends are visible in current camouflage design. One is towards "universal" designs (such as the Army Combat Uniform of the US Army), i.e. designs that perform well in a wide range of environments. Another trend is towards more specialized designs, such as a separate design for urban environments (e.g. the Canadian Urban Environment Pattern which is currently under investigation). Of course, a universal pattern will perform less well than a specialized pattern (see e.g. the very specialized patterns used for hunting). Since a universal pattern is probably not optimal the challenge is to find a design that is near-optimal in a variety of similar environments.

Since the urban environment differs largely from other theatres in their visual aspects as well as functional requirements a specialized urban camouflage design may perform much better than more traditional designs (created for other environments). Camouflage requirements for urban areas present a different challenge from those of natural environments like woodland or desert terrains. On the one hand, the tactical ranges are much closer in urban fighting than in woodland or desert warfare (over the last years the mean engagement distance has decreased to about $40 \mathrm{~m}$ ). This translates into designs with smaller macro patterns (i.e. patterns with closer merge distances). An assumption that is often used is that urban backgrounds require camouflage schemes with vertical and horizontal straight edges, and large macro patterns to mimic the pattern of the buildings and other objects found in an urban environment. Whereas natural environments display details at a wide range of scales (the spatial structure approximately independent of the viewing distance $^{5}$ ), man-made environments basically contain elements at two distinct scales (consisting of large structures and small details, and not much significant structures in between). As a result, disruptive patterns may be less effective, when viewed against contrasting areas of solid colour, like walls or pavement, and low contrast or uniform patterns may

*maarten.hogervorst@tno.nl; phone +31-346-356241; fax +31-346-353977

Infrared Imaging Systems: Design, Analysis, Modeling, and Testing XXI, edited by Gerald C. Holst, Keith A. Krapels, Proc. of SPIE Vol. 7662, 766205 - (c) 2010 SPIE · CCC code: 0277-786X/10/\$18 · doi: 10.1117/12.850423 
perform better in urban environments. These assumptions about optimal urban camouflage were evaluated by comparing performance of camouflage patterns that differ widely in their structure.

We have developed a method for deriving a camouflage pattern from a set of background images. This method can be used to create specialized camouflage designs (e.g. for an urban environment) or "multi-theatre" designs (derived from a wide variety of backgrounds from different theatres). Here, we present this method for deriving a camouflage pattern from a set of characteristic background images. As part of a NATO study (NATO SCI-157), we applied and evaluated this method to background images taken from the small town (arid climate) to create a design especially suited for this urban environment. In an evaluation study our design was compared with ten other designs which were especially designed for this environment. The various designs were made into prototype camouflage suits. We will discuss two experiments in which camouflage performance of the various camouflage designs was assessed.

\section{CAMOUFLAGE DESIGN METHOD}

We will give a global description of our method for deriving a camouflage pattern from a set of (characteristic) background images. Crucial parameters in the design are the chosen texture and colours.

\subsection{Texture}

Investigations into the textural properties of natural images show that natural images generally contain elements of all sizes and that the larger sized elements occur less often. Natural images show the same statistical behaviour as fractals (the power in spatial frequency decreases with one over the frequency ${ }^{5}$ ). Fractals are images in which the appearance essentially doesn't change when zooming in. We choose to use a similar texture, with a fractal nature. The advantage of such a texture is that it contains elements of various sizes. Therefore, for each background pattern there will be an element size in the pattern that matches the background elements. Also, the apparent size (as seen by the observer) will depend on the distances from the observer to the camouflage pattern and to the background pattern. For instance, when a camouflaged person stands right in front of a brick wall the best camouflage pattern will contain bricks of the same size as the bricks in the wall. When the brick wall is more in the distance, the bricks in the camouflage pattern should be smaller. By choosing a fractal pattern there are always elements in the pattern that are of the right size.

Most camouflage patterns contain a dominant direction, i.e. contain elements that are elongated. In case the background consists of the ground surface the person is standing on this makes sense (e.g. the perspective effect makes circular elements on the ground appear to be ellipses). However, backgrounds often contain objects blocking the view (e.g. trees, houses). Furthermore, when a person is lying down his/her orientation is changed and the pattern would no longer match the background. Therefore, we choose to use a pattern without dominant direction.



Figure 1. Illustration of the fact that camouflage patterns containing more detailed structure generally perform better. 


\subsection{Colour}

The colours were derived from a set of characteristic background samples (see Figure 2) and applied to a coloured fractal texture pattern which was designed such that the power spectrum in spatial frequency in the Hue, Saturation and Value signals falls of with one over the spatial frequency (1/f).



Figure 2 Image containing representative background samples, used for deriving the colours of the camouflage pattern.

A collage of characteristic background samples was made (see Figure 2). The colour distribution of this collage was transformed to the coloured fractal texture pattern as follows. First, both the background sample image and the texture were transformed to an indexed image $e^{\#}$ using 16 colours and standard indexing techniques. The 16 colours in the colour map of the coloured fractal as well as the collage were ordered in accordance of occurrence. Next, the colours in the coloured fractal (index image containing 16 colours) were replaced by the colours of the colour map belonging to the collage, to create a texture with a colour distribution that is similar to that of the collage. In the last step this pattern was approximated by a pattern containing the 4 prescribed colours (by NATO SCI-157). This was done by transforming the coloured texture (containing 16 colours) to RGB and then transforming it to an indexed image containing the 4 prescribed colours using standard dithering techniques.

\section{EVALUATION}

Recently, a NATO group investigated the effectiveness of camouflage uniforms in an urban environment. They implemented and assessed different methods for the design, fabrication, and evaluation of urban camouflage for the individual soldier system. A number of camouflage suits (see Figure 3) was designed based on imagery taken in a given (semi-arid) urban area. The suits included designs with micro-patterns that were synthesized using different techniques (numbers 4 through 10), suits especially designed to disrupt shapes (numbers 2 and 3), a monotone (sandy) suit (number 1) and an in-service (semi-arid) suit (number 11), an especially dark suit (number 10) and an especially light suit (number 4). We ran two experiments to evaluate the 11 different camouflage designs, see Figure 3 . In the first experiment camouflage performance was determined in detail at 2 locations. In the second experiment a human search experiment was performed using a large number of (36) locations.

\footnotetext{
\# In an indexed image the pixels do not contain RGB-values but entries (values ranging from 0 to 15 in this case) to a colour lookup table containing RGB-values.
} 


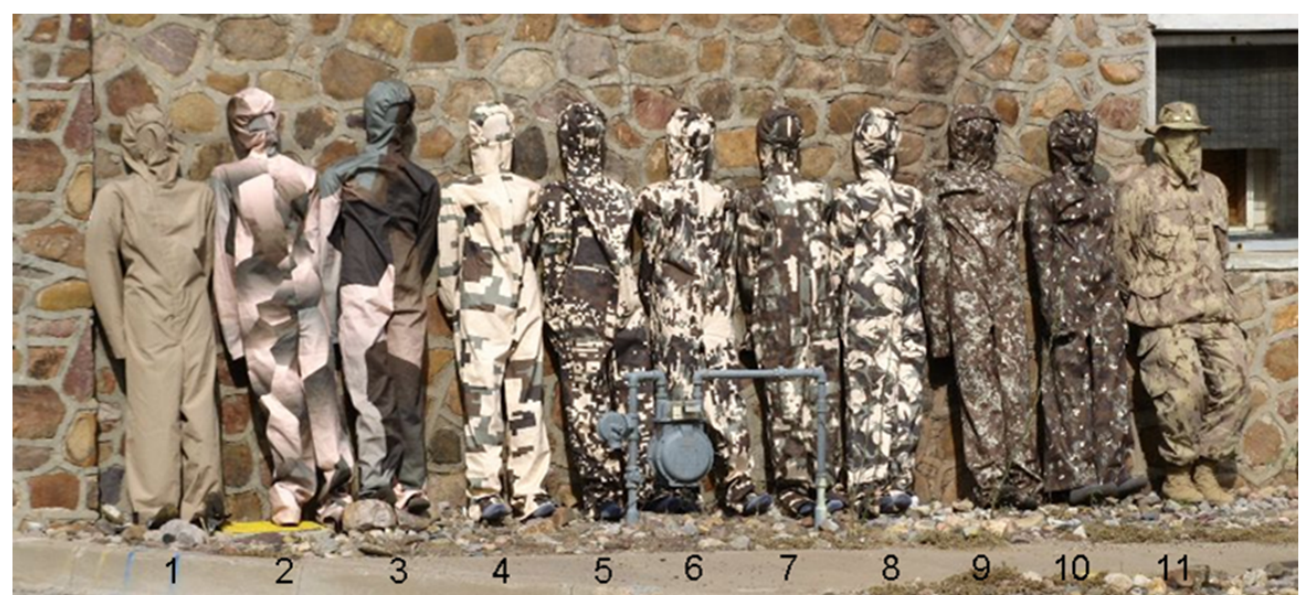

Figure 3 The 11 different suits that were evaluated. Suit number 9 is our design.

\subsection{Saliency experiment}

In the first experiment camouflage performance is assessed by investigating how well one can discriminate the target when fixating away from the target. The method used here is directly related to target conspicuity, which is found to be directly related to search performance ${ }^{6-8}$.

\section{Method}

Images were taken of persons wearing the 11 different camouflage suits on two locations (referred to as "shade" and "sun"), see Figure 4. The camouflaged persons were extracted from the images and pasted into a picture without target (camouflaged person). This was done to prevent recognition of the picture based on differences in areas outside the target.

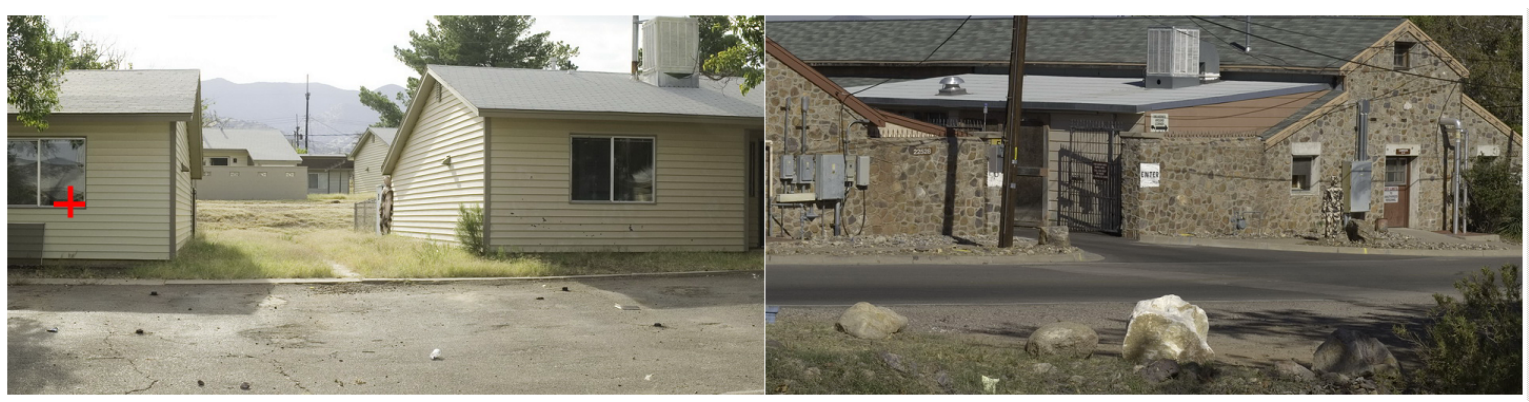

Figure 4. Images of the two locations used in the saliency experiment, referred to as "shade" (on the left) and "sun" (on the right).

A CRT-PC-monitor $(1600 \times 1200$ pixels $=40 \times 30 \mathrm{~cm}, 75 \mathrm{~Hz})$ was used and was viewed from a distance of about $40 \mathrm{~cm}$ with two eyes. The subject fixated a cross in the image next to one of the (left or right) edges (see e.g. cross in Figure 4a). In a given session the fixation cross was held on a fixed position on the screen. Each trial started by showing a mask image which was a pixelated version of the image containing no target. Then, in succession, an image with and without a target was shown in random order (i.e. first image could contain a target or not) separated by the mask image. The images were shown for $500 \mathrm{~ms}$ and the mask was shown for $1000 \mathrm{~ms}$. The task of the subject was to fixate the cross, and indicate afterwards which of the two images contained the target by clicking the mouse one or two times (a so-called $2 \mathrm{AFC}=$ two-alternative forced choice procedure was used).

In each session 4 different distances between fixation cross and target were used. For the "shade" image distances of $700,900,1100$ and 1300 pixels were used, while for the "sun" image distances between fixation cross and target of 300, 500,700 and 900 pixels were used. In each session 4 eccentricities were used (distances between fixation and target), 11 suits and 5 repetitions summing to a total of 220 trials per session. Each subject participated in 4 sessions per location. In the shade condition, in 2 of the sessions the fixation was on the left of the target, in the other 2 sessions fixation was right 
of the target. In the sun condition, fixation was to the left of the target in all 4 sessions. Four subjects (S1, S2, S3, S4) participated in the trials.

Results

Figure 5 shows the mean scores (average fraction correct, averaged over eccentricities) for all camouflage designs for the "sun" and the "shade" conditions, as well as the average over both conditions. The scores of the four subjects show a similar variation over designs indicating that some suits perform significantly better or worse than the others. Note that a low score indicates high camouflage performance. Some designs perform well in one location and poorly in the other location. This is illustrated in Figure 6 which shows the average fraction correct in the "sun" and in the "shade" for the different camouflage designs. The relatively bright designs 4 and 8 perform well (low fraction correct) in the "sun", but much less well in the "shade". Designs 11 and 1 perform well in the "shade", but much less well in the "sun" condition. Design 9 performs about equally well in both locations. 



Figure 5. Mean scores (averaged over all eccentricities) for all camouflage designs for the "sun" condition, the "shade" condition and the average over both locations. Shown are the scores of the individual subjects (S1..S4) as well as the average over all subjects. 


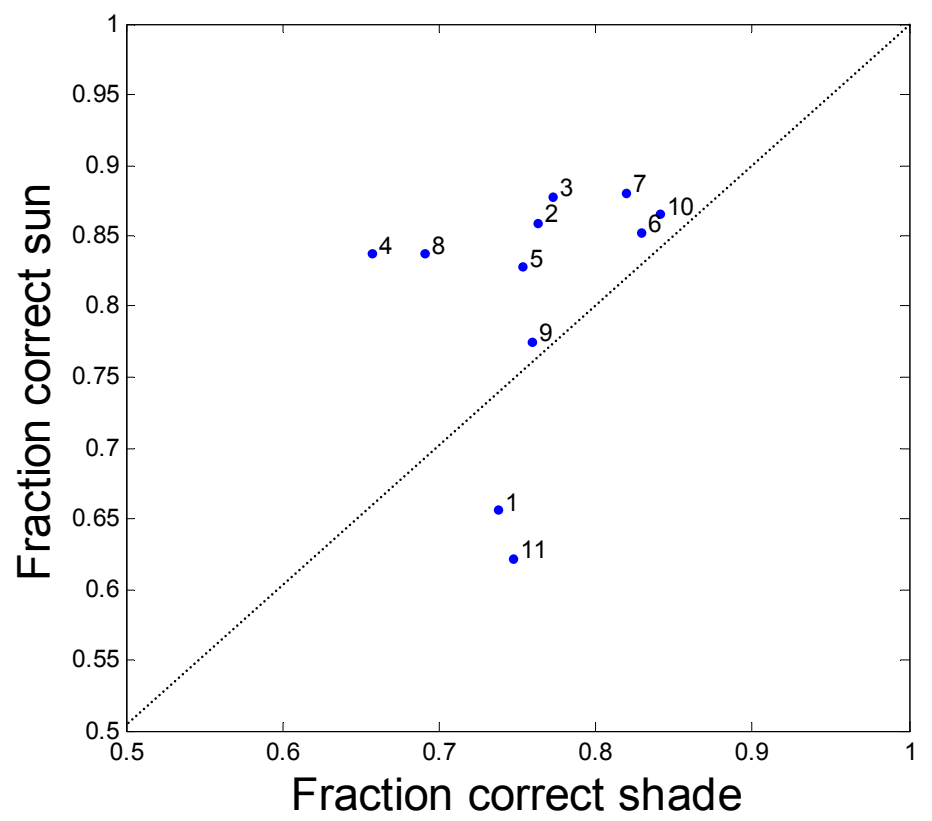

Figure 6. Average fraction correct (average over all subjects) in the "shade" and in the "sun" condition for the different camouflage designs.



Figure 7. Average score (average over both locations) of the different camouflage designs, in which the designs are ordered according to average score. Order numbers 1 to 11 correspond to target numbers: 11, 1, 4, 8, 9, 5, 2, 3, 6, 7, 10.

Figure 7 shows a version of the bottom graph of Figure 5 in which the suits are ordered such that the average score increases with order number. The ordered target numbers are: $11,1,4,8,9,5,2,3,6,7,10$. In these locations designs 11,1 and 4 perform relatively well whereas designs 6,7 and 10 show relatively poor camouflage performance. However, 
also clear is that there are large variations in performance over variations in backgrounds. A more suitable test of overall camouflage performance therefore includes more locations. Such a test is performed in experiment 2.

\subsection{Search experiment}

\section{Method}

Panorama images were used of a person wearing the 11 different camouflage suits (see Figure 3) on 36 locations (including the locations "sun" and "shade" analyzed in experiment 1). Eleven subjects participated in this experiment. The images were shown on a PC monitor (a 30" LCD screen with resolution of 2560x1600 pixels). In each trial a panorama image was shown containing a target or no target. The task of the subject was to decide whether the image contained a target and indicate as quickly as possible the target location by clicking the mouse on the target as soon as the target was detected. When the subject did not find a target he/she could indicate that no target was present by clicking on a square in the bottom-right corner of the screen. Each subject participated in one session in which 36 images with target were shown and 36 images without target. The subject was informed that there was a $50 \%$ chance that an image contained a target. Of either the image with the target or the image without target a mirror image was shown, to prevent possible recognition of the scene. The targets that were shown in a session were randomly picked from the set, such that all combinations of targets and target locations were shown to (combined) the set of 11 subjects.

\section{Results}

We found a low False alarm rate of about 7\%. More interesting are the hit rates of the different targets. Figure 8 a shows the average hit rate (i.e. the fraction of detected targets averaged over all locations and subjects) for the different camouflage suits. Figure $8 \mathrm{~b}$ show the same results with the suits ordered according to increasing hit rate. Note that a higher hit rate corresponds to poorer camouflage performance. This means that the suits with numbers $9,11,1$ and 8 perform relatively well, while suits with numbers $2,3,4$ and 7 perform relatively poor.
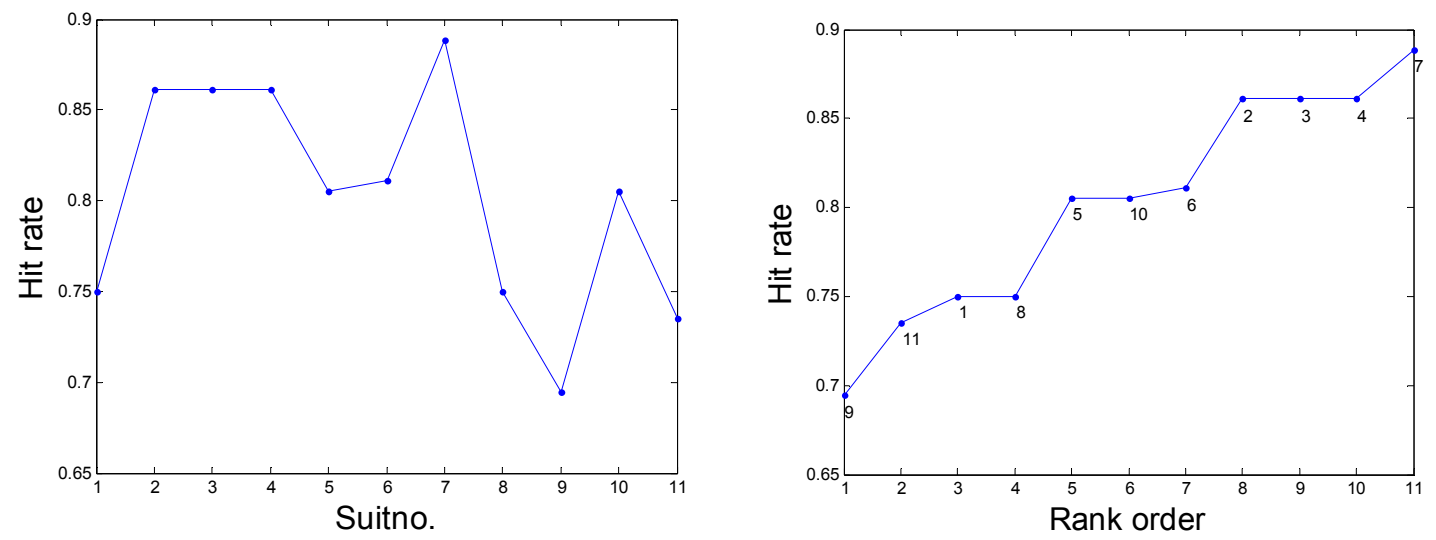

Figure 8. Average hit rate (average over all locations and subjects) of the different camouflage designs, a) hit rate as a function of suit number, b) hit rate of suits with increasing hit rate (suits ordered according to hit rate).

Another indication of camouflage performance is the time it takes to find a target. We therefore analyzed the trials in which the target was found. Figure 9 shows the average detection time of the various camouflage designs, in order of suit number and ordered according to (decreasing) detection time. Using this measure of performance, suits with numbers 11, 1 and 8 perform well and suits with numbers 3,4 and 2 perform (relatively) poor. 

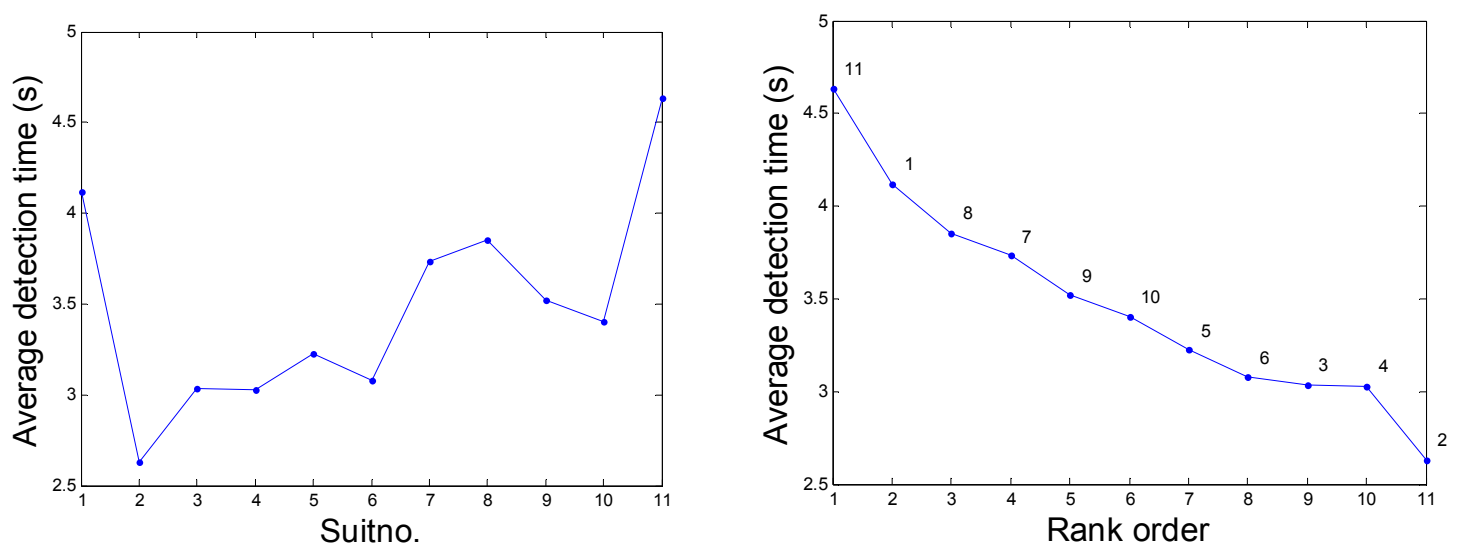

Figure 9. Average detection time of the different camouflage designs, a) as a function of suit number, ordered with decreasing detection time hit rate. The averages represent geometric means (averaged log-values) over trials in which the target was found, over all locations and subjects.

Both measures of performance are combined in Figure 10, which shows the average detection time and the hit rate of the different suits. Good camouflage performance is characterized by low hit rates and high average detection times (topright corner of Figure 10). Suits with numbers 9, 11, 1, 8 perform relatively well, whereas suits with numbers 2, 3, 4, 7 perform relatively poor.

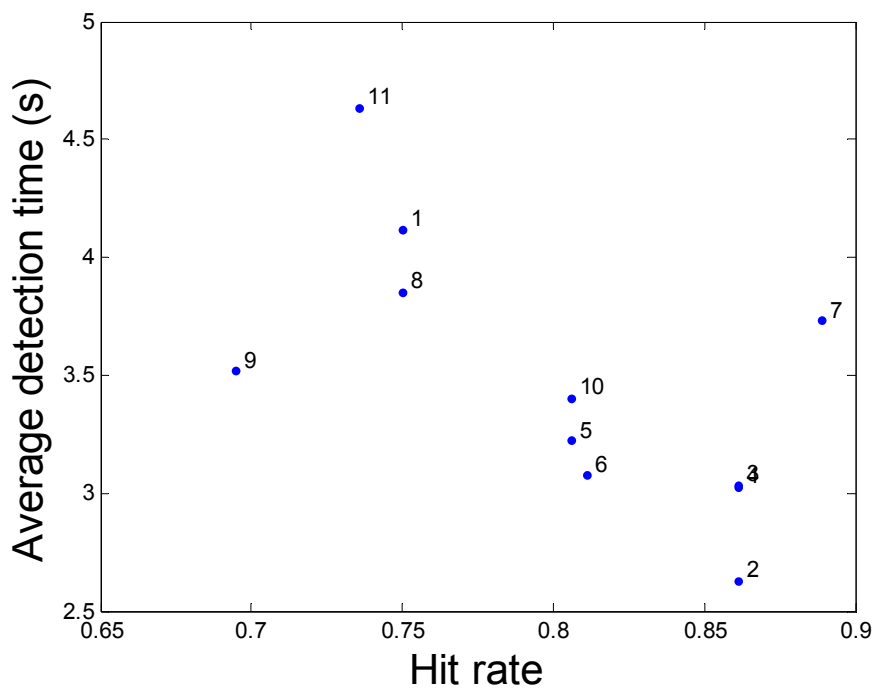

Figure 10. Average detection time vs. hit rate of the different camouflage designs. The average detection time represents the geometric mean (averaged log-values) over trials in which the target was found, over all locations and subjects.

The overall score attributed to the different suits will depend on the weight given to the two measures, although it seems obvious that the weight attributed to the hit rate should be relatively high. This will determine whether suit number 11 (with a high average detection time) or suit number 9 (with a low hit rate) will be attributed the highest score. Given the variability over different backgrounds (and over trials and subjects), it is well possible (see Figure 11) to discriminate high performing suits $(9,11,1,8)$ from medium $(10,5,6)$ and low performing suits $(2,3,4,7)$, but not to single out certain suits as the best performing suit (the standard error in the mean hit rate is around 0.05). More imagery taken at more locations (and more subjects) is required to allow a more detailed rank ordering of the camouflage designs. 



Figure 11. Overview of the overall camouflage performance of the various suits. Suits numbered 9, 11, 1, 8 (top row) show high performance, suits with numbers 10,5, 6 show medium performance (middle row) and suits numbered 7, 3, 4 and 2 show low performance (bottom row).

\section{CONCLUSION}

In this study 11 camouflage patterns were especially designed for a particular (arid) urban environment by different parties from different countries. One of the designs was based on a method we introduced. Here, we present our method for deriving camouflage patterns from background images, and we applied this method to images taken in this environment. Camouflage performance in this environment of all 11 designs was assessed by using panoramic 
photographs. In the first experiment camouflage performance was assessed in two locations using a method related to conspicuity. The second experiment involved a human search experiment using the panoramic image of all 11 suits in 36 locations. The camouflage performance measure obtained in experiment 1 is directly related to conspicuity, which is found to be a strong indicator of search performance. Suits 11 and 1 showed the best performance in these locations. However, the experiment also showed that camouflage performance can vary considerably over different locations. Therefore, a better indication of overall camouflage performance comes from experiments (such as experiment 2) in which more locations are considered. Measures of camouflage performance used in the second experiment are the fraction of locations in which the target was detected and the average search time over trials in which the targets were detected. The experiment allows us to distinguish good performing suits $(9,11,1,8)$ from medium $(10,5,6)$ or poorly performing suits $(7,3,4,2)$. The suit based on the design method presented here (suit number 9) shows good performance, indicating that our design method works quite well.

Interestingly, the assumptions that are often made regarding the best performing urban camouflage pattern were not confirmed in this study. Performance of the shape disruption patterns was not found to outperform patterns containing smaller scales. Also, the assumption that a good urban pattern should contain vertical and horizontal elements was not confirmed. This also questions the need for a camouflage pattern customized for a particular urban environment. Instead, our study suggests that general purpose arid patterns may provide good camouflage also in urban arid environments.

As mentioned in the introduction, one trend is towards the use of "universal" camouflage designs that perform well across a range of environments. Another possible direction may be opposite to this trend, i.e. heading towards camouflage patterns that are especially designed for particular environmental conditions. Algorithms (such as ours) to derive a camouflage pattern from a set of background images may be used to create such specialized patterns. New printing techniques that are currently under development, such as ink jet printing, may allow for rapid prototyping and low cost printing in the foreseeable future.

\section{REFERENCES}

1. Thayer, A. H. (1896) The law which underlies protective coloration. The Auk 13, 477-482.

2. Thayer, G. H. (1909) Concealing-coloration in the animal kingdom: an exposition of the laws of disguise through color and pattern: being a summary of Abbott H. Thayer's discoveries. New York, NY: Macmillan.

3. Cott, H. B. (1940) Adaptive coloration in animals. London, UK: Methuen \& Co. Ltd.

4. Troscianko, T., Benton, C. P., Lovell, P. G., Tolhurst, D. J. \& Pizlo, Z. (2009) Camouflage and visual perception.Phil.Trans. R. Soc. B 364, 449-461.

5. Tolhurst, D.J., Tadmor, Y. \& Chao, T. (1992) Amplitude spectra of natural images. Opthalmic and Physiological Optics, 12(2), 229-232.

6. Toet, A., Kooi, F.L., Bijl, P. \& Valeton, J.M. (1998) Visual conspicuity determines human target acquisition performance. Opt. Eng. 37, 1969-1975.

7. Toet, A. (2001) Visual conspicuity of targets in synthetic IR imagery. Toet, A. Camouflage, Concealment and Deception Evaluation Techniques. RTO-TM-017 AC/323(SCI-012)TP/32, 137-152. 2001. Neuilly-sur-Seine Cedex, France, North Atlantic Treaty Organization.

8. Toet, A. \& Bijl, P. (2003) Visual conspicuity. Driggers, R. G. Encyclopedia of optical engineering. 2929-2935. New York, USA, Marcel Dekker Inc. 